\title{
Changes observed in radionuclide bone scans during and after teriparatide treatment for osteoporosis
}

\author{
Amelia E. B. Moore • Glen M. Blake • \\ Kathleen A. Taylor • Valerie A. Ruff • Asad E. Rana • \\ Xiaohai Wan • Ignac Fogelman
}

Received: 20 June 2011 / Accepted: 12 October 2011 /Published online: 5 November 2011

(C) The Author(s) 2011. This article is published with open access at Springerlink.com

\begin{abstract}
Purpose Visual changes on radionuclide bone scans have been reported with teriparatide treatment. To assess this, serial studies were evaluated and quantified in ten postmenopausal women with osteoporosis treated with teriparatide $\left(20 \mu \mathrm{g}\right.$ /day subcutaneous) who had ${ }^{99 \mathrm{~m}} \mathrm{Tc}$-methylene diphosphonate (MDP) bone scans (baseline, 3 and 18 months, then after 6 months off therapy).

Methods Women were injected with $600 \mathrm{MBq}{ }^{99 \mathrm{~m}} \mathrm{Tc}-\mathrm{MDP}$, and diagnostic bone scan images were assessed at $3.5 \mathrm{~h}$. Additional whole-body scans (10 min, 1, 2, 3 and $4 \mathrm{~h}$ ) were analysed for ${ }^{99 \mathrm{~m}} \mathrm{Tc}-\mathrm{MDP}$ skeletal plasma clearance $\left(\mathrm{K}_{\mathrm{bone}}\right)$. Regional $\mathrm{K}_{\mathrm{bone}}$ differences were obtained for the whole skeleton and six regions (calvarium, mandible, spine, pelvis, upper and lower extremities). Bone turnover markers (BTM) were also measured.

Results Most subjects showed visual changes on 3- and 18month bone scan images that disappeared after 6 months off therapy. Enhanced uptake was seen predominantly in the calvarium and lower extremities. Whole skeleton $\mathrm{K}_{\mathrm{bone}}$ displayed a median increase of 22\% ( 3 months, $p=0.004$ ) and $34 \%$ (18 months, $p=0.002$ ) decreasing to $0.7 \%$ ( 6 months off therapy). Calvarium $K_{\text {bone }}$ changes were three times larger than other sites. After 6 months off therapy, all $\mathrm{K}_{\text {bone }}$ and BTM values returned towards baseline.
\end{abstract}

A. E. B. Moore $(\bowtie) \cdot$ G. M. Blake $\cdot$ I. Fogelman Department of Nuclear Medicine, Borough Wing; Guy's Hospital, King's College London School of Medicine,

St. Thomas Street,

London SE1 9RT, UK

e-mail: amelia.moore@kcl.ac.uk

K. A. Taylor · V. A. Ruff · A. E. Rana $\cdot$ X. Wan

Eli Lilly and Company and/or one of its subsidiaries,

Indianapolis, IN 46285, USA
Conclusion The increased ${ }^{99 \mathrm{~m}} \mathrm{Tc}-\mathrm{MDP}$ skeletal uptake with teriparatide indicated increased bone formation which was supported by BTM increases. After 6 months off therapy, metabolic activity diminished towards baseline. The modulation of ${ }^{99 \mathrm{~m}}$ Tc-MDP skeletal uptake during treatment was the result of teriparatide's metabolic activity. These findings may aid the radiological evaluation of similar teriparatide patients having radionuclide bone scans.

Keywords Teriparatide $\cdot{ }^{99 \mathrm{~m}} \mathrm{Tc}-\mathrm{MDP}$ bone scan $\cdot$ Bone turnover markers · Bone remodelling · Osteoporosis

\section{Introduction}

Radionuclide bone scan imaging using the radiopharmaceutical ${ }^{99 \mathrm{~m}} \mathrm{Tc}-$-methylene diphosphonate (MDP) has a useful role in both clinical practice and research to investigate metabolic bone diseases and response to treatment [1-3]. In clinical practice, bone scans are reported solely on a qualitative visual interpretation of images allowing for the detection of discrete changes in the skeleton in areas of focal tracer uptake. In the research setting, methods have been developed to translate visual assessments into quantitative data [4-7]. Skeletal plasma clearance of ${ }^{99 \mathrm{~m}} \mathrm{Tc}-\mathrm{MDP}\left[\mathrm{K}_{\text {bone }}(\mathrm{ml} / \mathrm{min})\right]$ measures the bone metabolic activity taking into account both the amount of radionuclide tracer cleared from plasma into bone and the blood input function. This quantitative measurement can be used to study the whole skeleton and regions of interest (ROI) [7].

Osteoporosis is a common skeletal disorder characterized by compromised bone strength predisposing a person to increased risk of fracture [8]. In the USA, 10 million individuals are estimated to have the disease, with 
osteoporosis responsible for approximately 1.5 million fractures annually [9]. Recombinant human parathyroid hormone fragment rhPTH(1-34) (teriparatide) is an anabolic therapy approved for use in women with postmenopausal osteoporosis, men with primary or hypogonadal osteoporosis, and patients with osteoporosis associated with sustained, systemic glucocorticoid therapy, all of whom are at high risk for fracture [10-12]. Teriparatide preferentially stimulates osteoblast over osteoclast activity resulting in new bone formation and an increase in the rate of bone remodelling. These effects manifest as an increase in skeletal mass, bone mineral density (BMD) and bone strength, subsequently leading to a reduction in the risk of fractures [13-19]. While it has been well established that teriparatide increases bone remodelling in the skeleton through histomorphometry studies $[18,20,21]$ and measurements of global bone turnover markers (BTM) [13, 17, 19], direct measurements of the effect of teriparatide on the metabolic activity of the skeleton have not been performed. Furthermore, questions have been raised by physicians regarding scintigraphic changes observed in patients taking teriparatide who have subsequently undergone radionuclide bone scan studies to evaluate other potential skeletal conditions [22], including a case of Paget's disease of bone [23].

This analysis evaluated the visual changes in the wholebody ${ }^{99 \mathrm{~m}} \mathrm{Tc}-\mathrm{MDP}$ bone scan images in ten subjects who had bone scans at baseline, 3 and 18 months of treatment with teriparatide, and after 6 months off therapy. We also measured levels of BTM and the skeletal plasma clearance of ${ }^{99 \mathrm{~m}} \mathrm{Tc}-\mathrm{MDP}$ in the whole skeleton and six ROI (calvarium, mandible, spine, pelvis, and upper and lower extremities) on treatment and after 6 months withdrawal from teriparatide.

\section{Materials and methods}

\section{Study population}

Ten postmenopausal Caucasian women with osteoporosis were treated with teriparatide $20 \mu \mathrm{g}$ /day by subcutaneous self-injection for 18 months followed by 6 months off therapy. A description of the subjects in this prospective, exploratory, Phase 4, open-label study was previously published [24]. Briefly, women were required to be between 50 and 85 years old and to have a total hip, femoral neck or lumbar spine BMD T-score $\leq-2.5$ or $<-2.0$ if they had a history of vertebral or non-vertebral fracture [25]. Hip and lumbar spine T-scores were calculated using the Third National Health and Nutrition Examination Survey (NHANES III) reference range [26] and the manufacturer's reference range (Hologic Inc., Bedford,
MA, USA), respectively. Subjects were excluded from the trial if they had a bone disorder, other than osteoporosis, that affected bone metabolism or if they had significant exposure to bone-active therapies prior to enrolment. For 2 months prior to the baseline visit and until the 24-month visit, study participants received daily supplements of calcium $(1,000 \mathrm{mg})$ and vitamin D (400-1,200 IU). The trial was conducted in accordance with good clinical practices and the Declaration of Helsinki. The local Ethics Committee approved the study and all women gave written informed consent.

Quantitative measurements of bone metabolism using ${ }^{99 \mathrm{~m}}$ Tc-MDP

Subjects underwent gamma camera bone scan imaging at baseline, 3 and 18 months after starting teriparatide therapy, and 6 months after stopping treatment. A dualhead gamma camera (ADAC Forte, ADAC Laboratories, Milpitas, CA, USA) was used to acquire anterior and posterior whole-body images with a scan speed of $25 \mathrm{~cm} /$ min at 10 min and at 1,2,3 and $4 \mathrm{~h}$ following intravenous injection of $600 \mathrm{MBq}{ }^{99 \mathrm{~m}} \mathrm{Tc}-\mathrm{MDP}$. For visual assessment of the bone scan images, a standard diagnostic scan (scan speed $10 \mathrm{~cm} / \mathrm{min}$ ) was performed at $3.5 \mathrm{~h}$. Subjects were advised to be well hydrated and, with the exception of the 10-min images, to empty their bladders before each wholebody scan. Upon completion of the 1-, 2-, 3- and 4-h scans, 5 -min right and left lateral spot views of the skull were acquired.

The method used to quantify the bone scan images was described previously [24]. After correction for background, urine activity in the bladder and kidneys, and radioactive decay, the whole-body retention of ${ }^{99 \mathrm{~m}} \mathrm{Tc}$ MDP was measured from the geometric mean of anterior and posterior total body counts by normalizing to the whole-body count (including bladder and kidneys) at $10 \mathrm{~min}$, which was defined as $100 \%$ [7]. Four regional sites (spine, pelvis, upper and lower extremities) were also analysed by copying ROIs defined on the 4-h anterior and posterior images (Fig. 1a) onto the 10-min and 1-, 2- and 3-h whole-body images [7]. The geometric mean of anterior and posterior counts was normalized to the 10 min whole-body count to express the results as the percentage of injected activity.

For the calvarium and mandible, the retention of ${ }^{99 \mathrm{~m}} \mathrm{Tc}$ MDP was measured by drawing appropriate ROI on the lateral images of the skull obtained at $4 \mathrm{~h}$ (Fig. 1b). These ROI were copied onto the 1-, 2- and 3-h images, and, after correction for background counts and radioactive decay, the geometric mean of the counts in the 1-, 2-, 3- and 4$\mathrm{h}$ images were normalized to the geometric mean of the counts from a point source of known activity measured 


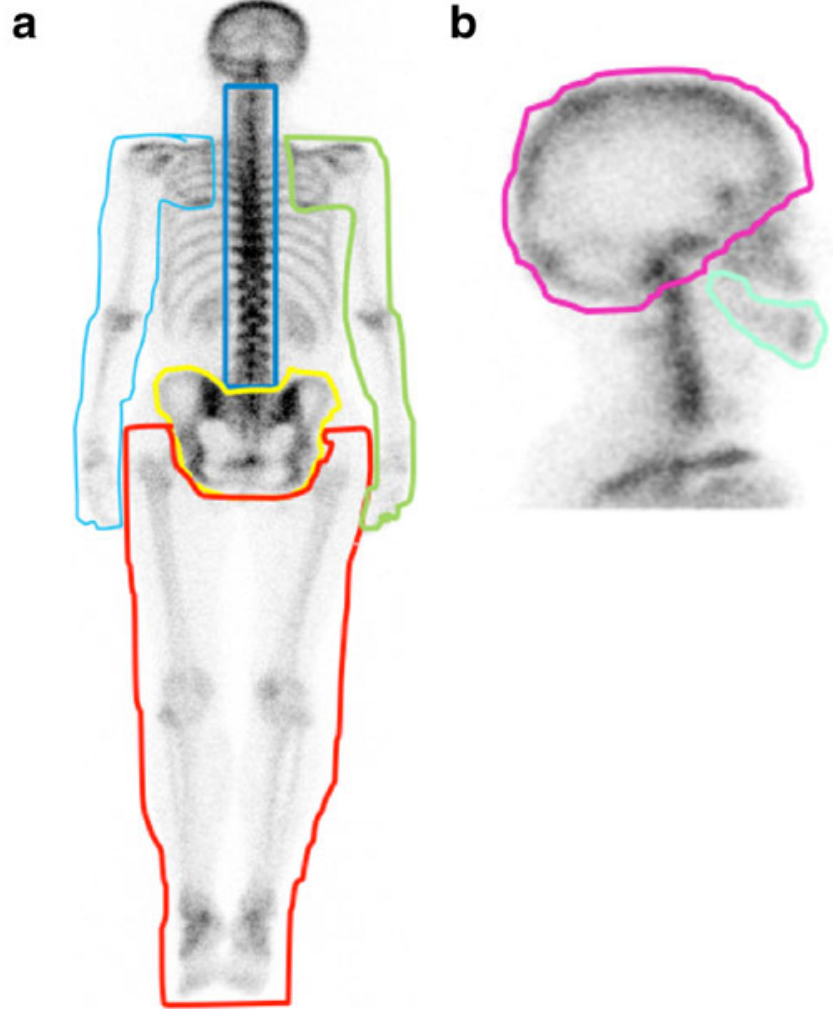

Fig. 1 ROI for the measurement of $\mathrm{K}_{\text {bone }}$ values for: a spine, pelvis, upper extremities and lower extremities. For the upper extremities the counts from the right and left arms were added together; $\mathbf{b}$ calvarium and mandible

with and without transmission through the skull to derive absolute uptake [27].

The ${ }^{99 \mathrm{~m}} \mathrm{Tc}-\mathrm{MDP}$ plasma time-activity curve was measured by taking blood samples at 5 and $20 \mathrm{~min}$ and 1, 2, 3 and $4 \mathrm{~h}$ after injection via an indwelling venous cannula in the opposite arm to the injection site. After blood sample centrifugation, $2 \mathrm{ml}$ of plasma was placed in 10-kDa filters (Amicon Ultra-4, Millipore Corp., Bedford, MA, USA) and spun for $30 \mathrm{~min}$ at 2,000 $\mathrm{g}$ [28]. An automatic gamma counter was used to determine the plasma concentration of free and total ${ }^{99 \mathrm{~m}} \mathrm{Tc}-\mathrm{MDP}$ in $1-\mathrm{ml}$ aliquots of ultrafiltrate and whole plasma.

Whole skeleton $\mathrm{K}_{\mathrm{bone}}$ and regional values of $\mathrm{K}_{\mathrm{bone}}$ for the calvarium, mandible, spine, pelvis, and upper and lower extremities were derived using the Patlak plot method [24]. Figures for percentage ${ }^{99 \mathrm{~m}}$ Tc-MDP uptake (bone plus soft tissue) in each ROI divided by the plasma concentration $\left(\% \mathrm{ml}^{-1}\right)$ of total (free plus bound) ${ }^{99 \mathrm{~m}} \mathrm{Tc}$ MDP were plotted on the vertical axis, and figures for the area under the plasma clearance curve (AUC) for free ${ }^{99 \mathrm{~m}}$ Tc-MDP divided by the total ${ }^{99 \mathrm{~m}}$ Tc-MDP plasma concentration were plotted on the horizontal axis. $\mathrm{K}_{\text {bone }}$ $\left(\mathrm{ml} \mathrm{min}{ }^{-1}\right)$ was derived from the slope of the straight line fitted to the 2-, 3- and 4-h data points [7].
Qualitative visual scoring of the ${ }^{99 \mathrm{~m}} \mathrm{Tc}-\mathrm{MDP}$ bone scan images

Qualitative visual scoring of changes in the diagnostic bone scan images at all four visits was performed by three reviewers who jointly classified the changes in whole skeleton or a regional site into the following groups as compared to baseline: (1) possible decreased uptake, (2) no discernible change in uptake, (3) possible increased uptake and (4) definite increased uptake.

BMD measurements and biochemical markers of bone turnover

BMD in the lumbar spine (L1-L4) and left hip was measured at baseline and 18 months using dual-energy Xray absorptiometry (DXA) (Hologic Inc., Bedford, MA, USA). The long-term precision was $1.6 \%$ for both spine and total hip BMD [29].

Fasting concentrations of BTM were measured at baseline and 3, 12, 18 and 24 months. The bone formation markers were serum procollagen type I N-terminal propeptide [PINP, Orion Diagnostica, Espoo, Finland; interassay coefficient of variance (CV) 3.1-8.2\%] and serum bonespecific alkaline phosphatase (BSAP, Hybritech, Beckman Coulter, Brea, CA, USA; interassay CV 7.4-7.9\%). The bone resorption marker was urinary excretion of $\mathrm{N}$-terminal telopeptide (NTX, Ostex, Seattle, WA USA; interassay CV $6.7-14.8 \%$ ) normalized to creatinine. The reference ranges for the BTM in postmenopausal women were specified by the central laboratory (Covance CLS, Geneva, Switzerland), which analysed the samples.

\section{Statistical analysis}

The Wilcoxon signed rank test was used to evaluate the per cent changes from baseline in $\mathrm{K}_{\mathrm{bone}}, \mathrm{BTM}$ and BMD. Due to the small sample size $(n=10)$, the changes were plotted as the median and interquartile range (IQR). The same test was used to evaluate the changes in $\mathrm{K}_{\text {bone }}$ and BTM during the 6-month period after stopping teriparatide therapy. Statistical inferences were made based on a two-sided significance level of 0.05 .

\section{Clinical laboratory and safety assessments}

At screening, parathyroid hormone $[\mathrm{PTH}(1-84)]$, 25hydroxyvitamin D, 24-h urine calcium and clinical chemistry were collected as previously described [24]. Measurements of serum calcium, alkaline phosphatase (AP) and creatinine were repeated at each subsequent visit. Adverse events were also assessed at each clinic visit. 


\section{Results}

Study population

The mean age of subjects was 68.3 years $( \pm 8.0)$. On average, subjects had severe osteoporosis with prevalent vertebral fractures and a mean ( \pm standard deviation) lumbar spine Tscore of $-3.2 \pm 0.8$ (Table 1). Clinical laboratory findings were within the normal reference range at baseline. BTM were within the reference ranges for postmenopausal women. All ten subjects returned for the 3- and 18-month scans and nine subjects returned for the 24-month visit.

Quantitative measurements of bone metabolism using ${ }^{99 \mathrm{~m}}$ Tc-MDP

After 3 months of teriparatide therapy, the median per cent increase from baseline in whole skeleton $\mathrm{K}_{\text {bone }}$ was $22.3 \%$ $(p=0.004)$, after 18 months the increase was $33.8 \%$ ( $p=$ $0.002)$, decreasing to $0.7 \%$ compared to baseline $(p=0.57)$ after 6 months off therapy (Fig. 2a). The decrease after stopping therapy was statistically significant $(p=0.004)$. In subjects with a history of prior osteoporotic fracture, there was negligible effect on global or regional skeletal uptake of ${ }^{99 \mathrm{~m}} \mathrm{Tc}-\mathrm{MDP}$ to interfere with the quantitative measurements [24]. The changes in $K_{\text {bone }}$ values for the regional sites are shown in Fig. $2 \mathrm{~b}-\mathrm{f}$. After 3 months of teriparatide treatment, the median increases from baseline in regional $\mathrm{K}_{\text {bone }}$ values were statistically significant in each of the six

Table 1 Baseline characteristics of the study population $(n=10)$

\begin{tabular}{lll}
\hline Characteristic & Mean $\pm \mathrm{SD}$ & Reference range $^{\mathrm{a}}$ \\
\hline Age (years) & $68.3 \pm 8.0$ & - \\
Years postmenopausal & $21.4 \pm 7.4$ & - \\
Body mass index $\left(\mathrm{kg} / \mathrm{m}^{2}\right)$ & $24.3 \pm 5.7$ & - \\
Lumbar spine T-score & $-3.2 \pm 0.8$ & - \\
Femoral neck T-score & $-2.5 \pm 0.8$ & - \\
BSAP $(\mu \mathrm{g} / \mathrm{l})$ & $12.5 \pm 3.6$ & $3.8-22.6$ \\
PINP $(\mu \mathrm{g} / \mathrm{l})$ & $38.9 \pm 13.1$ & $19.0-84.0$ \\
uNTx/Cr $(\mathrm{nmol} \mathrm{BCE} / \mathrm{mmol} \mathrm{Cr})$ & $29.0 \pm 19.0$ & $0-130$ \\
Serum creatinine $(\mu \mathrm{mol} / \mathrm{l})$ & $72.8 \pm 6.7$ & $31-101$ \\
Serum calcium $(\mathrm{mmol} / \mathrm{l})$ & $2.5 \pm 0.1$ & $2.1-2.6$ \\
Intact PTH $(1-84)(\mathrm{pmol} / \mathrm{l})$ & $3.1 \pm 1.3$ & $0.6-4.2$ \\
Serum AP $(\mathrm{U} / \mathrm{l})$ & $69.0 \pm 15.0$ & $35-123$ \\
\hline
\end{tabular}

BSAP bone-specific alkaline phosphatase, PINP N-terminal propeptide of collagen type I, $u N T x / C r$ urinary $\mathrm{N}$-telopeptide normalized to creatinine, $B C E$ bone collagen equivalent

${ }^{a}$ Reference ranges were provided by Covance Laboratories (Geneva, Switzerland), which analysed the samples. Ranges for BSAP, uNTx/ $\mathrm{Cr}$ and AP are for postmenopausal women; all others are for adult females sites studied: calvarium $72.3 \%$, mandible $65.9 \%$ (data not shown), spine $17.3 \%$, pelvis $20.3 \%$, upper extremities $42.5 \%$ and lower extremities $21.0 \%$. After 18 months of teriparatide treatment, the changes from baseline were statistically significant for five sites: calvarium $128.4 \%$, mandible $61.0 \%$ (data not shown), spine $33.8 \%$, upper extremities $95.5 \%$ and lower extremities $34.9 \%$. The change at the pelvis did not reach statistical significance at 18 months. After 6 months off teriparatide treatment, the median change from baseline in all regional $K_{\text {bone }}$ values declined towards baseline. The $\mathrm{K}_{\text {bone }}$ values at the spine $(16.9 \%)$ and the upper extremities $(62.8 \%)$ remained statistically significantly above baseline at 24 months. The decrease in $\mathrm{K}_{\text {bone }}$ from 18 to 24 months was statistically significant only at the calvarium and lower extremities.

Qualitative visual scoring of the ${ }^{99 \mathrm{~m}} \mathrm{Tc}-\mathrm{MDP}$ bone scan images

Qualitative visual scoring of the bone scan images compared with baseline at 3 and 18 months showed increased skeletal uptake that varied within and between subjects, with the 18-month changes more marked than those at 3 months. Increased ${ }^{99 \mathrm{~m}} \mathrm{Tc}-\mathrm{MDP}$ uptake was frequently noted in the calvarium and to a lesser extent in the long bones of the lower extremities. In six subjects after 6 months off therapy, the uptake on the bone scan image was visually indistinguishable from baseline. Of the other three subjects, one subject showed a possible decrease in uptake below baseline, and two subjects still had either a possible or definite increase in uptake above baseline.

Figures 3, 4 and 5 show anterior and posterior views of whole-body gamma camera bone scans and right lateral skull views of three subjects who were chosen to illustrate the range of visual changes. Tables 2, 3 and 4 show the corresponding $\mathrm{K}_{\text {bone }}$ and BTM data. Figure 3 shows subject 1 , with the most pronounced visual changes in skeletal uptake of ${ }^{99 \mathrm{~m}} \mathrm{Tc}-\mathrm{MDP}$ in the whole skeleton that were particularly prominent in the calvarium and long bones of the legs. The values for whole skeleton $\mathrm{K}_{\text {bone, }}$ as well as each regional site, and all BTM at 3 and 18 months were substantially higher than the baseline values (Table 2) with whole skeleton $\mathrm{K}_{\text {bone }}$ increasing by 76 and $60 \%$ at 3 and 18 months, respectively, while $\mathrm{K}_{\text {bone }}$ for the calvarium increased by 152 and $188 \%$, respectively. This subject completed 18 months of teriparatide therapy, but was subsequently lost to follow-up.

Figure 4 shows subject 2, with a definite increase in skeletal uptake of ${ }^{99 \mathrm{~m}} \mathrm{Tc}-\mathrm{MDP}$ in the calvarium and possibly subtler visual changes in the rest of the skeleton compared to baseline at both 3 and 18 months. The changes observed in the calvarium on the whole-body bone scans are also supported by those seen in the lateral skull views. 

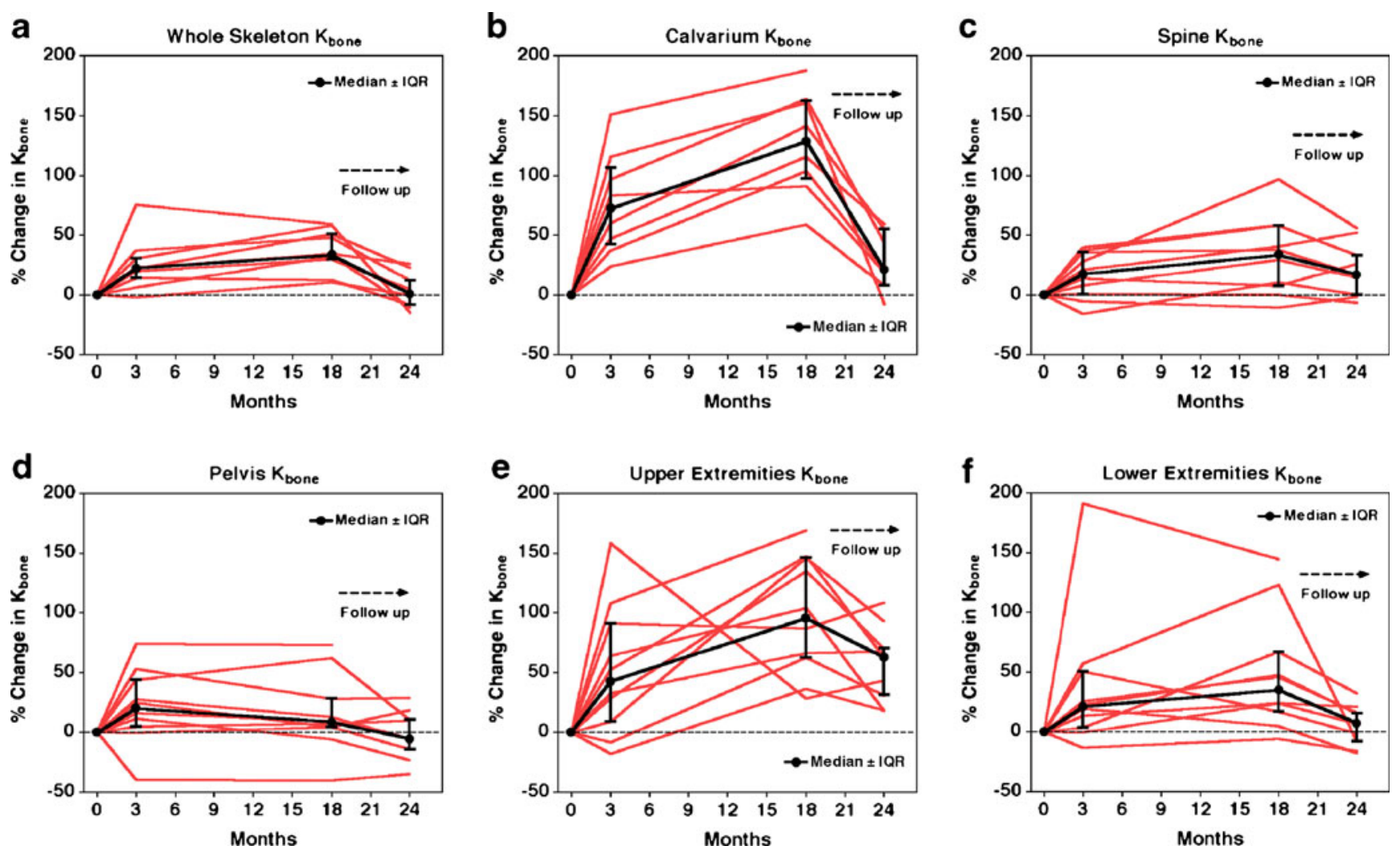

Fig. 2 Per cent changes from baseline of the $\mathrm{K}_{\text {bone }}$ values for the whole skeleton and five of the six skeletal regional sites studied after 3 and 18 months of teriparatide, followed by 6 months off therapy. Data are shown for all ten individual subjects (red lines) and the median (black line). Error bars show the IQR. Data for the mandible are not shown. a Whole skeleton $K_{\text {bone, }}$ b calvarium $K_{\text {bone, }}$ c spine $K_{\text {bone, }}$ d pelvis $\mathrm{K}_{\text {bone, }}$ e upper extremities $\mathrm{K}_{\text {bone, }} \mathbf{f}$ lower extremities $\mathrm{K}_{\text {bone. }}$ $K_{\text {bone }}{ }^{99 \mathrm{~m}} \mathrm{Tc}-\mathrm{MDP}$ skeletal plasma clearance, $I Q R$ interquartile range
At 24 months, the visual appearance of the whole-body scans and the lateral skull images returned to that observed at baseline. The percentage change from baseline for whole skeleton $\mathrm{K}_{\text {bone }}$ at 3 and 18 months for this subject was 23 and $33 \%$, respectively (Table 3), which is similar to the median per cent change observed for the study group. The percentage change from baseline in $\mathrm{K}_{\mathrm{bone}}$ for the calvarium at 3 and 18 months was 116 and $161 \%$, respectively, diminishing to below the baseline value at 24 months. Similarly, the $\mathrm{K}_{\text {bone }}$ value for whole skeleton returned to below the baseline value at 24 months.

Figure 5 shows subject 3 , with a possible increase in skeletal uptake in the calvarium at 3 months and a definite increase in skeletal uptake in this site at 18 months compared to the baseline image, with the lateral skull views supporting this interpretation. No clear visual changes are observed in the rest of the skeleton at 3 and 18 months. At 24 months, the visual appearance of the calvarium returned to that observed at the baseline visit. The percentage change from baseline for whole skeleton $\mathrm{K}_{\text {bone }}$ at 3 and 18 months for this subject was 20 and $30 \%$, respectively (Table 4), which is similar to the median per cent change observed for the study group. The percentage change in $\mathrm{K}_{\mathrm{bone}}$ for the calvarium between baseline, 3, 18 and 24 months was 37,104 and $21 \%$, respectively.

BMD measurements and biochemical markers of bone turnover

As reported previously, at 18 months the median percentage change from baseline in BMD at the lumbar spine and total hip was $8.7 \%(p=0.01)$ and $0.9 \%(p=0.30)$, respectively [24]. At 3, 12 and 18 months, subjects had statistically significant increases in concentrations of serum BSAP, PINP and urinary NTX/creatinine ratio, and after 6 months off therapy, all markers declined towards baseline (Fig. 6). The decrease in BTM from 18 to 24 months, representing 6 months off therapy, was statistically significant for BSAP $(p=0.008)$ and PINP $(p=0.004)$ but not for NTX $(p=0.91)$. Concentrations of serum AP were within the reference range at baseline (Table 1), modestly elevated by $16.2 \%$ at 12 months $(p=0.037)$ and returned to baseline at 24 months (Fig. 6d).

BSAP and PINP were statistically significantly correlated with whole skeleton $\mathrm{K}_{\text {bone }}$ values at 3 and 18 months (Spearman rank correlation coefficients 0.60 

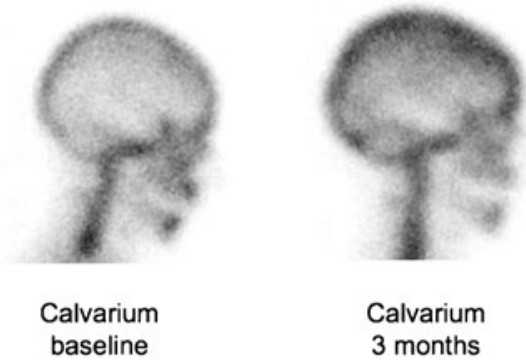

Calvarium 3 months

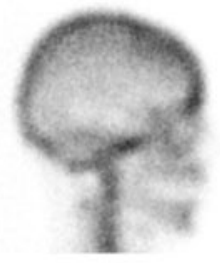

Calvarium 18 months
Baseline

3 months teriparatide

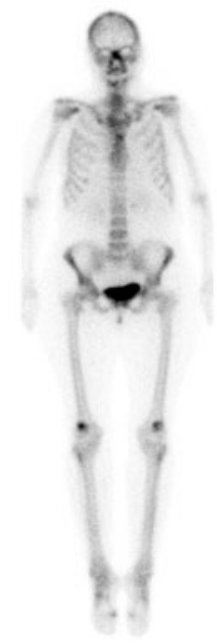

Anterior

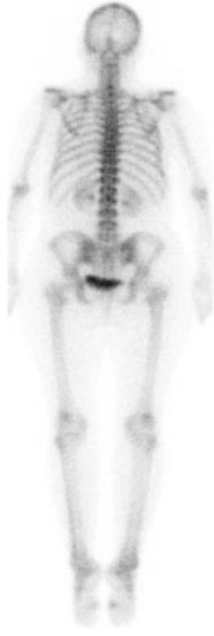

Posterior

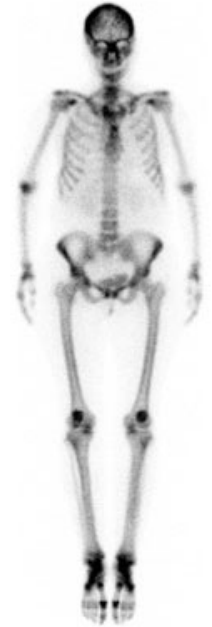

Anterior

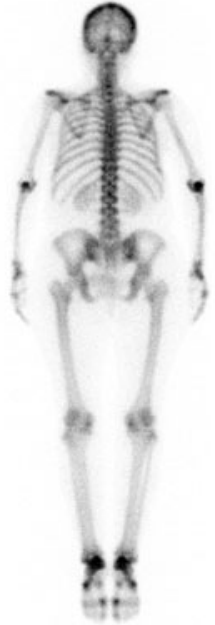

Posterior
18 months teriparatide

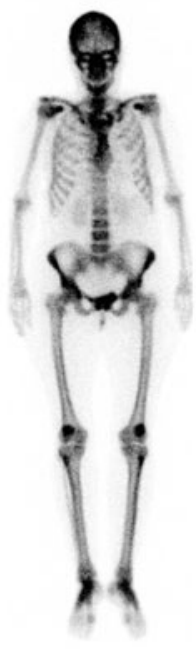

Anterior

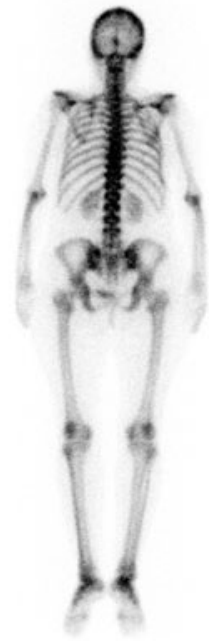

Posterior

Fig. $3{ }^{99 \mathrm{~m}} \mathrm{Tc}-\mathrm{MDP}$ anterior and posterior whole-body bone scans and right lateral skull views at baseline, 3 and 18 months for subject 1

and 0.78 , respectively, $p<0.05)$, as described in a previous report [24].

\section{Adverse events}

Teriparatide was well tolerated in the study group, with adverse events similar to those seen in larger clinical trials. Serious adverse events in subjects on therapy were few and reported previously [24]. During the 6 months off therapy, no serious adverse events were reported. Subject 1 reported randomly occurring mild joint/bone pain in the left leg after taking teriparatide for 2 months. This continued for 8 months while the subject was receiving teriparatide, but the severity did not increase or affect any other regions of the skeleton during this period. After resolution of the pain, the subject continued to receive teriparatide for a further 9 months without complaint.

\section{Discussion}

This is the first study to report the visual and quantitative changes measured by radionuclide bone scan in subjects undergoing 18 months of treatment with teriparatide followed by 6 months off therapy. Although quantitative changes at 3 and 18 months were previously described [24], the present analysis examined the variety of visual changes and compared these results to quantitative measures of $\mathrm{K}_{\text {bone }}$ and BTM. Furthermore, this report describes the effects of therapy withdrawal on qualitative and quantitative measures of skeletal metabolism.

The study showed that quantitative measurements of skeletal plasma clearance were a more robust indicator of the effects of treatment than visual changes on the bone scan images. After 3 and 18 months of teriparatide treatment, measurements of whole skeleton and regional $\mathrm{K}_{\text {bone }}$ were significantly increased compared with baseline, with the value nearly doubling in subject 1. Greater increases in $\mathrm{K}_{\text {bone }}$ were seen after 18 months of teriparatide treatment compared with 3 months for the whole skeleton and every regional site except the pelvis. The largest changes in $\mathrm{K}_{\text {bone }}$ were observed in the calvarium, which was also the site that showed the most pronounced visual changes. Six months after stopping teriparatide, whole skeleton and regional $\mathrm{K}_{\text {bone }}$ values returned toward baseline values, although the calvarium and lower extremities 

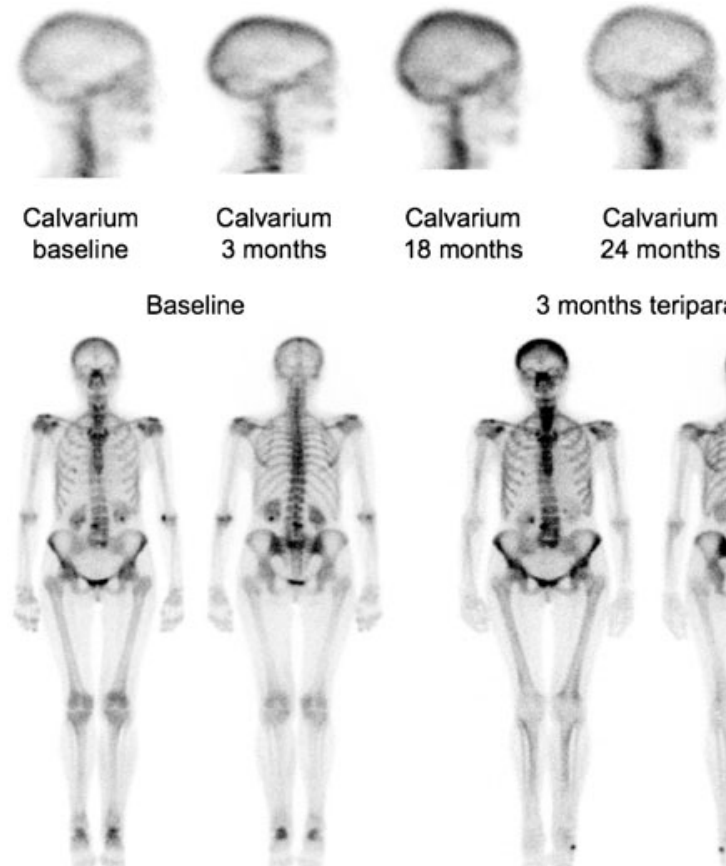

Calvarium 3 months

Calvarium

Calvarium

24 months 3 months teriparatide

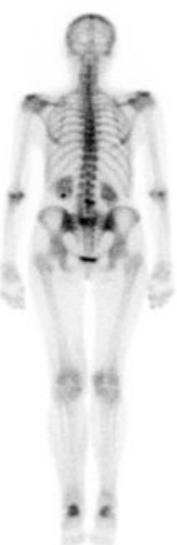

Anterior

Posterior

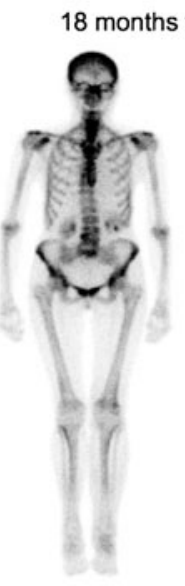

Anterior

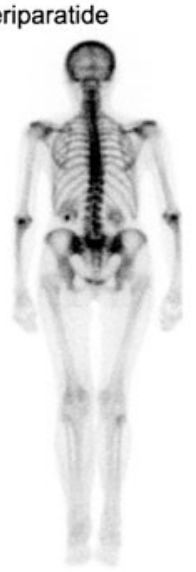

Posterior

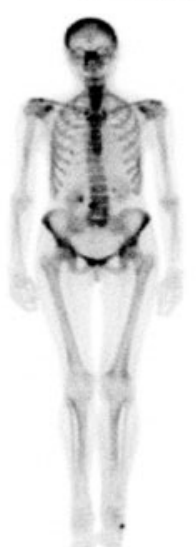

Anterior
6 months off teriparatide

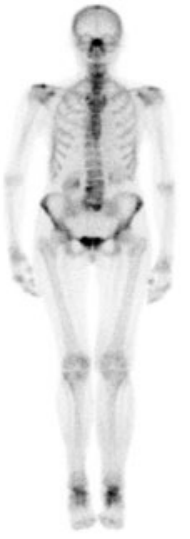

Anterior

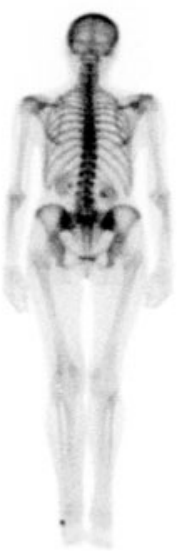

Posterior

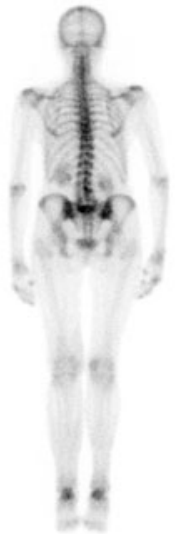

Fig. $4{ }^{99 \mathrm{~m}}$ Tc-MDP anterior and posterior whole-body bone scans and right lateral skull views at baseline, 3, 18 and 24 months for subject 2

remained somewhat elevated. However, at both of these sites there was a statistically significant decrease between 18 and 24 months.

One subject (number 1) showed particularly pronounced visual increases in ${ }^{99} \mathrm{~m}$ Tc-MDP uptake throughout the skeleton (Fig. 3). Of the other nine subjects, after 18 months of teriparatide treatment, seven showed a definite increase in uptake, one had possible increased uptake and one showed no increase at any site. The changes were most evident in the calvarium and, in some subjects, the long bones of the lower extremities, with the change in contrast between these areas and the rest of the skeleton providing the clearest visual evidence of the metabolic response to
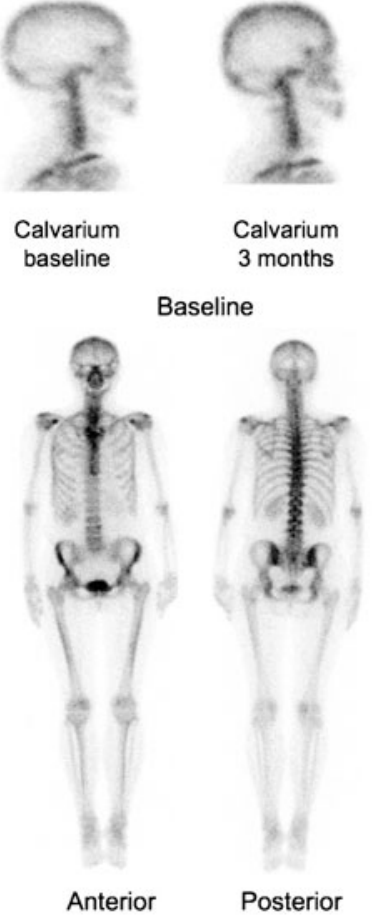

18 months teriparatide

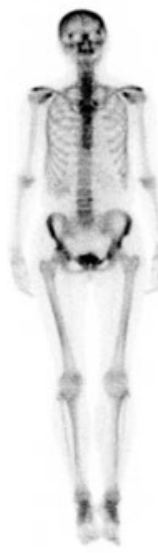

Anterior

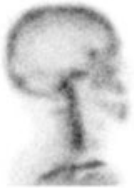

Calvarium

\section{riparatide}

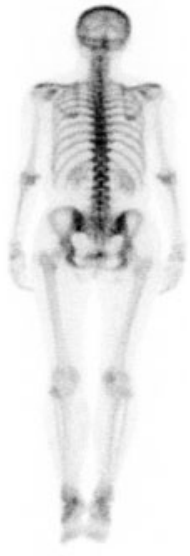

Posterior
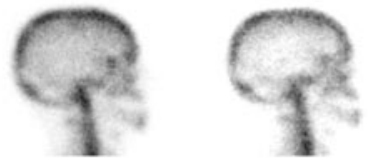
Calvarium Calvarium 18 months 24 months

3 months teriparatide

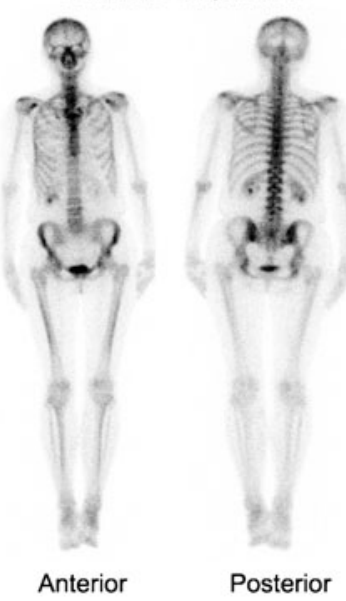

6 months off teriparatide
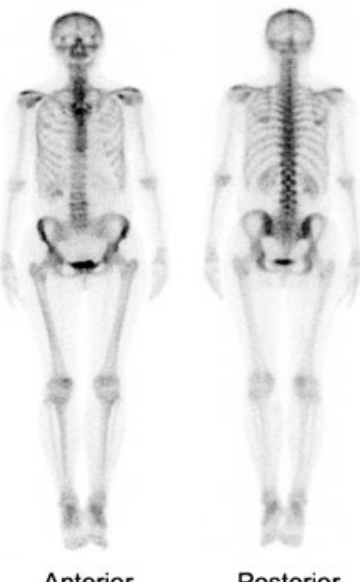

Posterior
Fig. $5{ }^{99 \mathrm{~m}}$ Tc-MDP anterior and posterior whole-body bone scans and right lateral skull views at baseline, 3, 18 and 24 months for subject 3

teriparatide treatment. Thus, the visual changes in bone scan images during treatment with teriparatide appeared to be variable and ranged from marked global increases in uptake, to increases in the calvarium and lower extremities, to increases in the calvarium alone, to no discernable change (Figs. 3, 4 and 5). As expected after 6 months off teriparatide therapy, the appearance of the bone scan images was visually indistinguishable from baseline in most subjects, which suggests that the metabolic changes in the skeleton are the result of the anabolic mechanism of action of teriparatide, and not due to other skeletal diseases.

In the literature, a case study has reported the possible development of Paget's disease of bone in the humerus and 
Table 2 Absolute values at baseline, 3 and 18 months for $\mathrm{K}_{\text {bone }}$ and bone turnover markers in subject 1
$K_{\text {bone }}{ }^{99 \mathrm{~m}} \mathrm{Tc}-\mathrm{MDP}$ skeletal plasma clearance, BSAP bonespecific alkaline phosphatase, PINP N-terminal propeptide of collagen type I, $u N T X / C r$ urinary N-telopeptide normalized to creatinine, $B C E$ bone collagen equivalent

\begin{tabular}{llll}
\hline Parameter & Baseline & 3 months & 18 months \\
\hline Kbone $(\mathrm{ml} / \mathrm{min})$ & & & \\
Whole skeleton & 42.5 & 74.6 & 67.8 \\
Calvarium & 3.2 & 8.1 & 9.3 \\
Mandible & 0.2 & 0.4 & 0.6 \\
Spine & 5.7 & 8.0 & 9.0 \\
Pelvis & 5.8 & 10.1 & 10.0 \\
Upper extremities & 3.7 & 7.6 & 9.8 \\
Lower extremities & 8.3 & 24.2 & 20.4 \\
Bone turnover markers & & & \\
BSAP $(\mu \mathrm{g} / \mathrm{l})$ & 7.5 & 15.5 & 14.4 \\
PINP $(\mu \mathrm{g} / \mathrm{l})$ & 38 & 216 & 190 \\
$\mathrm{uNTx} / \mathrm{Cr}(\mathrm{nmol}$ BCE/nmol Cr) & 20 & 169 & 202 \\
AP $(\mathrm{U} / \mathrm{l})$ & 47 & 71 & 71 \\
\hline
\end{tabular}

pelvis of a patient during treatment with teriparatide [23]. In addition, when some patients taking teriparatide underwent a bone scan to evaluate another medical condition, their whole-body bone scans showed increased uptake of tracer in the calvarium, which mimicked the appearance of Paget's disease of bone. In these reports, skeletal uptake was diminished but persisted for 3 months after therapy discontinuation. Since changes in ${ }^{99 m}$ Tc-MDP skeletal uptake lag behind changes in bone formation, the final bone scan in this study was obtained 6 months after stopping teriparatide, so that it was possible to detect a significant decrease in skeletal plasma clearance and study the attenuation of the teriparatide-induced anabolic activity. The pattern of tracer uptake observed with both visual and quantitative assessments returned towards baseline 6 months after stopping teriparatide therapy. This suggests that the changes observed in these subjects during treatment are the result of metabolic activity of the drug rather than a process of bone pathology. Given the reported metabolic changes induced by teriparatide treatment $[12,13]$ and the findings described here, it is not surprising that similar visual changes described previously have been attributed to bone disease in individual cases. Although these findings provide reference data for physicians when evaluating bone scan changes in patients taking teriparatide, the presence of underlying pathologies should be carefully considered in conjunction with patient history and medical status.

The changes in spine and hip BMD and BTM reported here are typical of those previously observed with teriparatide treatment [10-13]. Bone markers showed the greatest increase between baseline and 3 months, generally peaked at 12 months, and were unchanged or decreased at 18 months (Fig. 6). After 6 months off therapy, PINP and NTX were still significantly increased, although they had returned towards baseline values. Similar behaviour was seen with BSAP and AP.
Table 3 Absolute values at baseline, 3, 18 and 24 months for $\mathrm{K}_{\mathrm{bone}}$ and bone turnover markers in subject 2

$K_{\text {bone }}{ }^{99 \mathrm{~m}} \mathrm{Tc}-\mathrm{MDP}$ skeletal plasma clearance, BSAP bonespecific alkaline phosphatase, PINP N-terminal propeptide of collagen type I, $u N T X / C r$ urinary $\mathrm{N}$-telopeptide normalized to creatinine, $B C E$ bone collagen equivalent

\begin{tabular}{lllll}
\hline Parameter & Baseline & 3 months & 18 months & 24 months \\
\hline Kone $(\mathrm{ml} / \mathrm{min})$ & & & & \\
Whole skeleton & 37.2 & 45.9 & 49.5 & 33.2 \\
Calvarium & 2.9 & 6.3 & 7.7 & 2.7 \\
Mandible & 0.1 & 0.2 & 0.2 & 0.2 \\
Spine & 6.7 & 9.0 & 9.2 & 7.8 \\
Pelvis & 6.8 & 7.6 & 6.4 & 5.3 \\
Upper extremities & 5.2 & 8.6 & 10.7 & 6.2 \\
Lower extremities & 9.7 & 8.4 & 9.2 & 8.1 \\
Bone turnover markers & & & & \\
BSAP ( $\mu \mathrm{g} / \mathrm{l})$ & 19.2 & 15.5 & 18.0 & 14.3 \\
PINP $(\mu \mathrm{g} / \mathrm{l})$ & 57 & 136 & 149 & 48 \\
uNTx/Cr (nmol BCE/nmol Cr) & 61 & 77 & 41 & 65 \\
AP (U/l) & 81 & 72 & 73 & 66 \\
\hline
\end{tabular}


Table 4 Absolute values at baseline, 3, 18 and 24 months for $\mathrm{K}_{\mathrm{bone}}$ and bone turnover markers in subject 3
$K_{\text {bone }}{ }^{99 \mathrm{~m}} \mathrm{Tc}-\mathrm{MDP}$ skeletal plasma clearance, BSAP bonespecific alkaline phosphatase, PINP N-terminal propeptide of collagen type I, $u N T X / C r$ urinary $\mathrm{N}$-telopeptide normalized to creatinine, $B C E$ bone collagen equivalent

\begin{tabular}{lllll}
\hline Parameter & Baseline & 3 months & 18 months & 24 months \\
\hline Kbone $(\mathrm{ml} / \mathrm{min})$ & & & & \\
Whole skeleton & 32.7 & 39.3 & 42.5 & 34.2 \\
Calvarium & 2.8 & 3.9 & 5.8 & 3.4 \\
Mandible & 0.2 & 0.2 & 0.2 & 0.2 \\
Spine & 6.7 & 7.2 & 8.6 & 7.7 \\
Pelvis & 6.2 & 6.5 & 6.7 & 6.8 \\
Upper extremities & 3.5 & 6.7 & 6.5 & 7.3 \\
Lower extremities & 6.5 & 8.1 & 9.4 & 7.5 \\
Bone turnover markers & & & & \\
BSAP $(\mu \mathrm{g} / \mathrm{l})$ & 9.2 & 10.7 & 13.2 & 8.7 \\
PINP $(\mu \mathrm{g} / \mathrm{l})$ & 32 & 56 & 99 & 46 \\
uNTx/Cr (nmol BCE/nmol Cr) & 17 & 36 & 39 & 63 \\
AP (U/l) & 66 & 65 & 69 & \\
\hline
\end{tabular}

The most important limitation of this study was the small number of subjects. For this reason, it is unclear how frequently a bone scan showing the pronounced visual changes seen in Fig. 3 may occur as a result of teriparatide treatment, or whether a response in terms of BTMs can occur without a corresponding visual change in the bone scan. The subjects in this study were all Caucasian postmenopausal women, so information regarding the changes observed on bone scans in other populations treated with teriparatide is not available.

Nevertheless, this study has several strengths. Radionuclide bone scan images provide a unique way of visualizing and comparing the metabolic changes in bone in response to teriparatide therapy in different regions of the skeleton compared with BTM that show only the average integrated response. Quantitative measurements of skeletal plasma
Fig. 6 Per cent change from baseline of BTMs after 3, 12 and 18 months of teriparatide followed by 6 months off therapy. Data are shown for all ten individual subjects (red lines) and the median (black line). Error bars show the IQR. a BSAP, b PINP, c uNTX/Cr, d Alk Phos. Alk Phos alkaline phosphatase, BSAP serum bonespecific alkaline phosphatase, $B T M$ bone turnover markers, $I Q R$ interquartile range, $u N T X / C r$ urinary excretion of $\mathrm{N}$-terminal telopeptide normalized to creatinine, PINP serum procollagen type I N-terminal propeptide
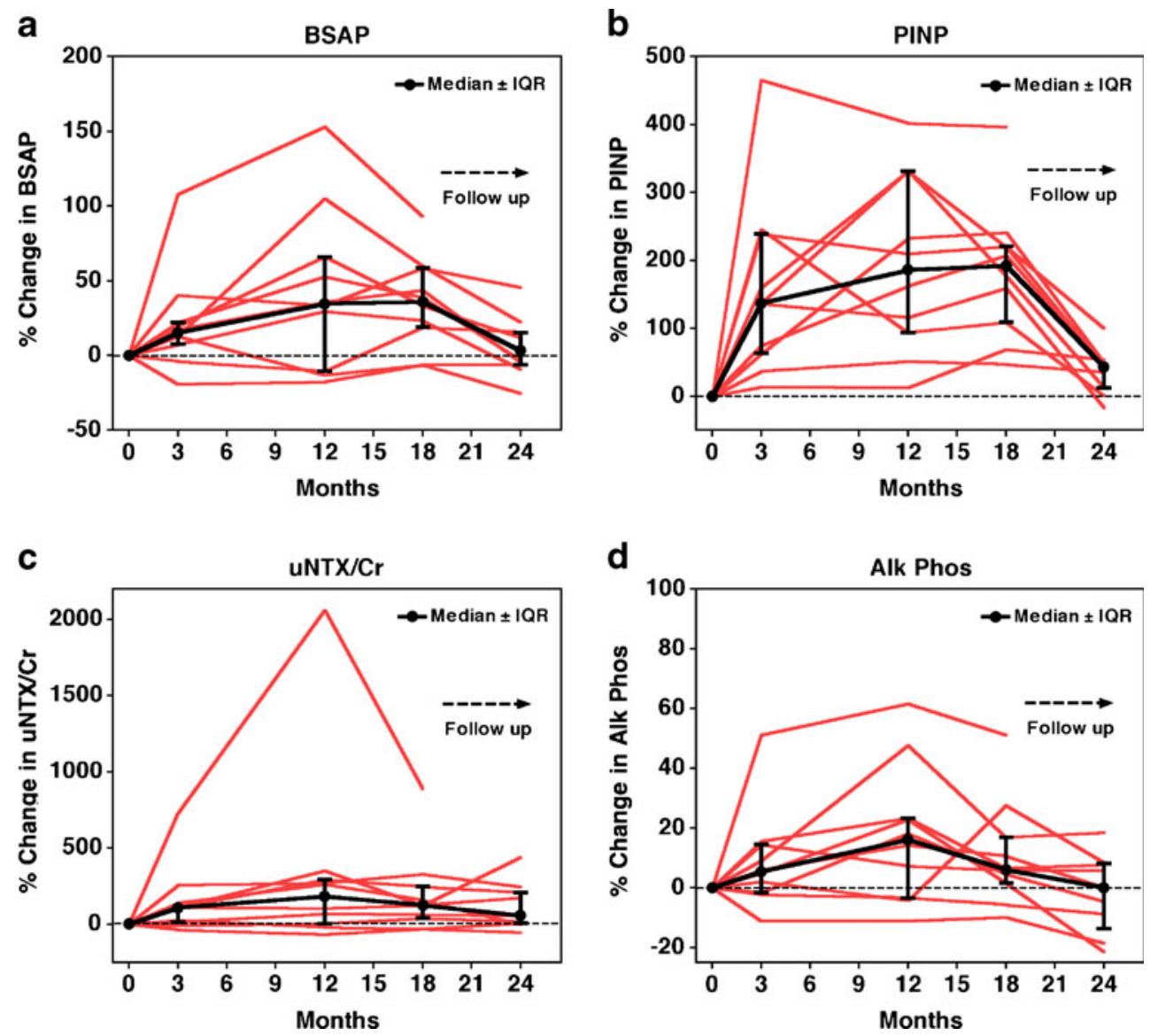

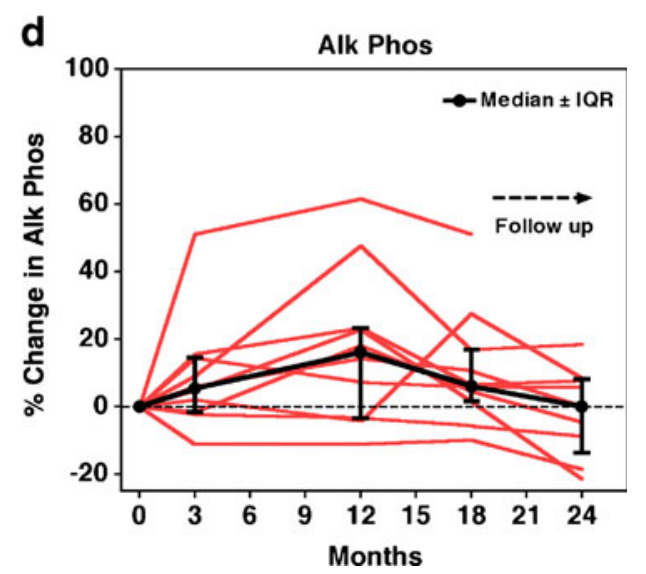


clearance provided a more sensitive indicator of response to treatment than visual assessments alone and were able to confirm the regional differences observed at sites of prominent visual changes such as the calvarium. This study provides additional data suggesting that the calvarium is particularly sensitive to the metabolic action of teriparatide, although further study is required to determine the exact nature of this response. Additionally, the concurrent assessment of radionuclide bone scans, BTM and BMD allowed for quantitative comparisons between the visual images and $\mathrm{K}_{\text {bone }}$ measurements with surrogate markers that show well-characterized responses to teriparatide treatment.

\section{Conclusion}

We have reported the visual changes in the radionuclide bone scan seen during teriparatide treatment and its subsequent withdrawal. Increased skeletal uptake of ${ }^{99 \mathrm{~m}} \mathrm{Tc}-\mathrm{MDP}$ was observed in postmenopausal women with osteoporosis treated with teriparatide, and the visual changes were confirmed by quantitative measurements of ${ }^{99 \mathrm{~m}}$ Tc-MDP skeletal plasma clearance and BTM. The changes were most frequently seen in the calvarium. After 6 months off teriparatide, the appearance of the bone scan images returned toward baseline, and this was accompanied by a decline in skeletal plasma clearance and BTM. The results of this study may offer practical information to aid the visual interpretation of bone scans in patients concurrently receiving teriparatide treatment.

Acknowledgments This work was sponsored by Lilly USA, LLC and/or Eli Lilly and Company. The authors acknowledge Yuqin Li for assistance with statistical analysis. Laura Bean Warner (Medical Writer, i3 Statprobe) and David Meats (Medical Writer, i3 Statprobe) are acknowledged for assistance with preparation of the manuscript.

Source of financial support This work was sponsored by Eli Lilly and Company and/or one of its subsidiaries.

Conflicts of interest Drs. Moore, Blake, and Fogelman received a research grant from Eli Lilly and Company as clinical trial investigators. Drs. Taylor, Rana, and Wan, and Ruff, MS are employees of Eli Lilly and company and/or one of its subsidiaries.

Open Access This article is distributed under the terms of the Creative Commons Attribution Noncommercial License which permits any noncommercial use, distribution, and reproduction in any medium, provided the original author(s) and source are credited.

\section{References}

1. Subramanian G, McAfee JG, Blair RJ, Kallfelz FA, Thomas FD. Technetium-99m-methylene diphosphonate-a superior agent for skeletal imaging: comparison with other technetium complexes. J Nucl Med 1975;16:744-55.

2. Gnanasegaran G, Cook GJ, Fogelman I. Radionuclide scintigraphy in metabolic bone disease. In: Rosen CJ, editor. Primer on the metabolic bone diseases and disorders of mineral metabolism. Washington: American Society for Bone and Mineral Research; 2008. p. 166-9.

3. Cook GJ, Gnanasegaran G, Chua S. Miscellaneous indications in bone scintigraphy: metabolic bone diseases and malignant bone tumors. Semin Nucl Med 2010;40:52-61.

4. Fogelman I, Bessent RG, Turner JG, Citrin DL, Boyle IT, Greig WR. The use of whole-body retention of Tc-99m diphosphonate in the diagnosis of metabolic bone disease. J Nucl Med 1978;19:270-5.

5. Brenner W, Bohuslavizki KH, Sieweke N, Tinnemeyer S, Clausen M, Henze E. Quantification of diphosphonate uptake based on conventional bone scanning. Eur J Nucl Med 1997;24:1284-90.

6. Scillitani A, Dicembrino F, Chiodini I, Minisola S, Fusilli S, Di Giorgio A, et al. Global skeletal uptake of $99 \mathrm{mTc}-m e t h y l e n e$ diphosphonate (GSU) in patients affected by endocrine diseases: comparison with biochemical markers of bone turnover. Osteoporos Int 2002;13:829-34.

7. Moore AE, Blake GM, Fogelman I. Quantitative measurements of bone remodeling using $99 \mathrm{mTc}-$ methylene diphosphonate bone scans and blood sampling. J Nucl Med 2008;49:375-82.

8. NIH Consensus Development Panel on Osteoporosis Prevention, Diagnosis, and Therapy. Osteoporosis prevention, diagnosis, and therapy. JAMA 2001;285:785-95.

9. Bone health and osteoporosis: a report of the Surgeon General. Available at: www.surgeongeneral.gov/library/bonehealth. Accessed 20 May 2011.

10. Neer RM, Arnaud CD, Zanchetta JR, Prince R, Gaich GA, Reginster JY, et al. Effect of parathyroid hormone (1-34) on fractures and bone mineral density in postmenopausal women with osteoporosis. N Engl J Med 2001;344:1434-41.

11. Orwoll ES, Scheele WH, Paul S, Adami S, Syversen U, DiezPerez A, et al. The effect of teriparatide [human parathyroid hormone (1-34)] therapy on bone density in men with osteoporosis. J Bone Miner Res 2003;18:9-17.

12. Saag KG, Shane E, Boonen S, Marín F, Donley DW, Taylor KA, et al. Teriparatide or alendronate in glucocorticoid-induced osteoporosis. N Engl J Med 2007;357:2028-39.

13. McClung MR, San Martin J, Miller PD, Civitelli R, Bandeira F, Omizo M, et al. Opposite bone remodeling effects of teriparatide and alendronate in increasing bone mass. Arch Intern Med 2005; 165:1762-8.

14. Chen P, Satterwhite JH, Licata AA, Lewiecki EM, Sipos AA, Misurski DM, et al. Early changes in biochemical markers of bone formation predict BMD response to teriparatide in postmenopausal women with osteoporosis. J Bone Miner Res 2005;20:962-70.

15. Chen P, Miller PD, Delmas PD, Misurski DA, Krege JH. Change in lumbar spine BMD and vertebral fracture risk reduction in teriparatide-treated postmenopausal women with osteoporosis. J Bone Miner Res 2006;21:1785-90.

16. Chen P, Miller PD, Recker R, Resch H, Ranan A, Pavo I, et al. Increases in BMD correlate with improvements in bone microarchitecture with teriparatide treatment in postmenopausal women with osteoporosis. J Bone Miner Res 2007;22:1173-80.

17. Dobnig H, Sipos A, Jiang Y, Fahrleitner-Pammer A, Ste-Marie LG, Gallagher JC, et al. Early changes in biochemical markers of bone formation correlate with improvements in bone structure during teriparatide therapy. J Clin Endocrinol Metab 2005;90:3970-7.

18. Jiang Y, Zhao JJ, Mitlak BH, Wang O, Genant HK, Eriksen EF. Recombinant human parathyroid hormone (1-34) [teriparatide] improves both cortical and cancellous bone structure. J Bone Miner Res 2003;18:1932-41. 
19. Glover SJ, Eastell R, McCloskey EV, Rogers A, Gamero P, Lowery J, et al. Rapid and robust response of biochemical markers of bone formation to teriparatide therapy. Bone 2009;45(6):1053-8.

20. Arlot M, Meunier PJ, Boivin G, Haddock L, Tamayo J, Correa-Rotter $\mathrm{R}$, et al. Differential effects of teriparatide and alendronate on bone remodeling in postmenopausal women assessed by histomorphometric parameters. J Bone Miner Res 2005;20:1244-53.

21. Ma YL, Zeng Q, Donley DW, Ste-Marie LG, Gallagher JC, Dalsky GP, et al. Teriparatide increases bone formation in modeling and remodeling osteons and enhances IGF-II immunoreactivity in postmenopausal women with osteoporosis. J Bone Miner Res 2006;21:855-64.

22. Lim GC, Wafa WS, Moses AM. Increased focal uptake of Tc-99m labelled MDP on bone scan suggestive of hyperostosis frontalis interna in a patient being treated with teriparatide. J Bone Miner Res 2005;20 Suppl 1:S174.

23. Mapa J, Conte S, Greene L. Teriparatide-induced Paget's disease of bone. J Bone Miner Res 2009;24 suppl 1:S464.

24. Moore AE, Blake GM, Taylor KA, Rana AE, Wong M, Chen P, et al. Assessment of regional changes in skeletal metabolism following 3 and 18 months of teriparatide treatment. J Bone Miner Res 2010;25:960-7.

25. World Health Organisation. Assessment of fracture risk and its application to screening for postmenopausal osteoporosis. Geneva, Switzerland: WHO; 1994. Technical report series no. 843 .

26. Looker AC, Wahner HW, Dunn WL, Calvo MS, Harris TB, Heyse SP, et al. Updated data on proximal femur bone mineral levels of US adults. Osteoporos Int 1998;8:468-89.

27. Jonsson L, Ljungberg M, Strand SE. Evaluation of accuracy in activity calculations for the conjugate view method from Monte Carlo simulated scintillation camera images using experimental data in an anthropomorphic phantom. J Nucl Med 2005;46:167986.

28. Moore AE, Hain SF, Blake GM, Fogelman I. Validation of ultrafiltration as a method of measuring free $99 \mathrm{mTc}-\mathrm{MDP}$. J Nucl Med 2003;44:891-7.

29. Patel R, Blake GM, Rymer J, Fogelman I. Long-term precision of DXA scanning assessed over seven years in forty postmenopausal women. Osteoporos Int 2000;11:68-75. 